\title{
An Update on the ACS Virgo and Fornax Cluster Surveys
}

\author{
Patrick Côté $^{1}$, Laura Ferrarese ${ }^{1}$, Andrés Jordán ${ }^{2}$, John P. Blakeslee ${ }^{3}$, \\ Chin-Wei Chen ${ }^{1,4}$, Leopoldo Infante ${ }^{5}$, Simona Mei ${ }^{6}$, Eric W. Peng ${ }^{1}$, \\ John L. Tonry ${ }^{7}$ and Michael J. West ${ }^{8}$ \\ ${ }^{1}$ Herzberg Institute of Astrophysics, Victoria, Canada; ${ }^{2}$ Harvard-Smithsonian Center for \\ Astrophysics, Cambridge, MA $;{ }^{3}$ Washington State University, Pullman, WA; ${ }^{4}$ National \\ Central University, Taiwan; ${ }^{5}$ Pontificia Universidad Católica de Chile, Chile; ${ }^{6}$ Observatoire de \\ Paris, France; ${ }^{7}$ Institute for Astronomy, Honolulu, USA $;{ }^{8}$ European Southern Observatory, \\ Santiago, Chile
}

\begin{abstract}
We present a brief update on the ACS Virgo and Fornax Cluster Surveys - Hubble Space Telescope programs to obtain ACS imaging for 143 early-type galaxies in the two galaxy clusters nearest to the Milky Way. We summarize a selection of science highlights from the surveys as including new results on the central structure of early-type galaxies, the apparent continuity of photometric and structural parameters between dwarf and giant galaxies, and the properties of globular clusters, diffuse star clusters and ultra-compact dwarf galaxies.
\end{abstract}

Keywords. galaxies: elliptical and lenticular, cD galaxies: fundamental parameters - galaxies: nuclei - galaxies: photometry - galaxies: star clusters - galaxies: structure

\section{The ACS Virgo and Fornax Cluster Surveys: An Introduction}

With the installation of the Advanced Camera for Surveys (ACS) in 2002, the already outstanding imaging capabilities of the Hubble Space Telescope (HST) improved dramatically. To capitalize on the roughly factor of ten improvement in "discovery efficiency" (Ford et al. 1998), our team initiated - in Cycles 11 and 13 - ACS surveys of the early-type galaxy populations in the Virgo and Fornax Clusters: the two rich clusters nearest to the Milky Way. These ACS Virgo and Fornax Cluster Surveys (ACSVCS, Côté et al. 2004; ACSFCS, Jordán et al. 2007a) consist of HST imaging for 143 members of the Virgo and Fornax Clusters, all of which were previously classified on the basis of ground-based photographic imaging to have early-type morphologies (e.g., types E, S0, dE, dE,N or dS0; Binggeli, Sandage \& Tammann 1985; Ferguson 1989). All ACS images were taken in WFC mode using a filter combination (F475W and F850LP) equivalent to the $g$ and $z$ bandpasses in the SDSS photometric system. The images cover a $200^{\prime \prime} \times 200^{\prime \prime}$ field with $\approx 0.1^{\prime \prime}$ resolution, corresponding to a physical resolution of $\approx 8-9 \mathrm{pc}$ at the distances of Virgo and Fornax (16.5 and $19.5 \mathrm{Mpc}$, respectively). The Virgo sample is complete for early-type galaxies brighter than $M_{B} \approx-19.2$ and $44 \%$ complete down to its limiting magnitude of $M_{B} \approx-15.2$, while the Fornax sample is complete down to $M_{B} \approx-16.1$. In addition to the ACS images that comprise the core datasets for both surveys, our group has obtained supplemental imaging and spectroscopy from WFPC2, Chandra, Spitzer, Keck, VLT, Magellan, KPNO, and CTIO.

The core science goals of the surveys include the measurement of luminosities, colors, metallicities, ages, and structural parameters for the many thousands of globular clusters associated with the early-type galaxies in these clusters, a high-resolution isophotal analysis of galaxies spanning a factor of $\approx 720$ in luminosity, and the measurement of 
accurate distances for the full sample of galaxies using the method of surface brightness fluctuations (SBF). In this article, we give a brief summary of selected scientific highlights as of September 2007.

\section{The Transition from Central Luminosity Deficit to Excess: No Core/Power-law Dichotomy}

Pioneering HST imaging studies of the centers of early-type galaxies suggested an apparently abrupt transition in central stellar density at $M_{B} \sim-20.3 \mathrm{mag}$ - the socalled "core/power-law dichotomy" (e.g., Ferrarese et al. 1994; Lauer et al. 1995). These findings prompted and supported the widely held view that the bright ("core") and faint ("power-law") galaxies follow distinct evolutionary routes (e.g., Ebisuzaki, Makino \& Okumura 1991; Faber et al. 1997). The evidence for such a dichotomy has lessened — although not entirely disappeared — following more recent studies that identified a population of galaxies with intermediate properties (Rest et al. 2001; Ravindranath et al. 2001).

By analyzing the ACS brightness profiles for the 100 ACSVCS galaxies, Ferrarese et al. (2006a) showed that Sérsic models provide more accurate parameterizations of the global brightness profiles than the so-called "Nuker models" (Lauer et al. 1995; Lauer et al. 2007) used in most previous analyses of HST brightness profiles (see also Graham et al. 2003; Graham 2004; Ferrarese et al. 2006c) and argued that the core/power-law dichotomy is an artifact introduced, in part, by the use of an inappropriate (i.e., powerlaw) parameterization of the outer profiles, combined with a tendency in previous work (which often relied on HST brightness profiles of limited radial extent) to not properly account for the compact stellar nuclei found in low- and intermediate luminosity galaxies (e.g., Graham \& Guzmán 2003; Grant, Kuipers \& Phillipps 2005; Côté et al. 2006).

For each ACSVCS and ACSFCS galaxy, azimuthally-averaged $g z$ surface brightness profiles were derived as described in Ferrarese et al. (2006a). As we have noted elsewhere (e.g., Ferrarese et al. 2006abc; Côté et al. 2006, 2007; Jordán et al. 2007a), there is systematic behavior of the brightness profiles on small scales (see the left panels of Fig. 1): galaxies brighter than $M_{B} \sim-20$ typically show central light deficits with respect to the inward extrapolation of the Sérsic (1968) model that best fits the profile at radii larger than $\sim 0.1^{\prime \prime}-1^{\prime \prime}$, while the great majority of low- and intermediate-luminosity galaxies $\left(-19.5 \lesssim M_{B} \lesssim-15\right)$ show central light excesses. Galaxies occupying a narrow range of intermediate luminosities $\left(-20 \lesssim M_{B} \lesssim-19.5\right)$ are reasonably well fitted by Sérsic models over all radii (see also Graham et al. 2003).

In light of this systematic behavior, and to provide a more robust characterization of the inner regions of galaxies, Côté et al. (2007) introduced a parameter, $\Delta_{0.02}=$ $\log \left(\mathcal{L}_{g} / \mathcal{L}_{s}\right)$ - where $\mathcal{L}_{g}$ and $\mathcal{L}_{s}$ are the integrated luminosities inside $0.02 R_{e}$ of the observed profile and of the inward extrapolation of the outer Sérsic (1968) model - to describe the central luminosity deficit $\left(\Delta_{0.02}<0\right)$ or excess $\left(\Delta_{0.02}>0\right)$. We find that $\Delta_{0.02}$ varies smoothly over the range of $\approx 720$ in blue luminosity spanned by the Virgo and Fornax sample galaxies, with no evidence for a dichotomy (see the right panel of Fig. 1). A review of the possible formation models for these central excesses was presented in Côté et al. (2006), including our favored interpretation (Côté et al. 2007) that they are the analogs of the "dense central cores" predicted by some numerical simulations to form as a result of gas inflows (see, e.g., Mihos \& Hernquist 1994). 

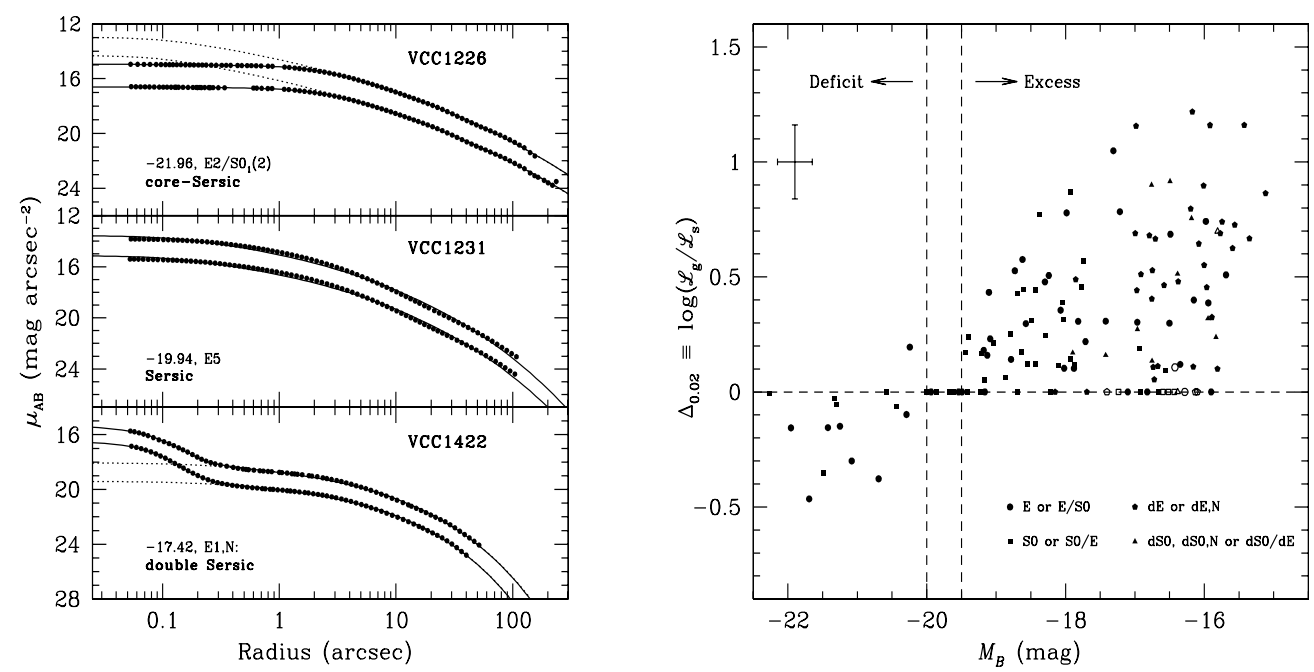

Figure 1. (Left Panels) Surface brightness profiles in $g(\mathrm{~F} 475 \mathrm{~W})$ and $z$ (F850LP) for three galaxies from the ACS Virgo Cluster Survey, illustrating the transition from central "deficit" to "excess" relative to the inward extrapolation of Sérsic models that fit the outer profiles (dotted curves). See $\S 2$ and $\S 3$ for details on the parameterization of the profiles. (Right) Dependence of $\Delta_{0.02}=\log \left(\mathcal{L}_{g} / \mathcal{L}_{s}\right)$ on galaxy magnitude for ACSVCS and ACSFCS galaxies. Galaxies with central luminosity deficits have $\Delta_{0.02}<0$ while those with central excesses have $\Delta_{0.02}>0$. Note the smooth transition from central deficit to excess with decreasing galaxy luminosity. A typical errorbar is shown on the left side of the panel. Open symbols in this plot denote galaxies with "dE/dIrr transition" morphologies, dust, young stellar clusters and/or evidence of young stellar populations from blue integrated colors.

\section{A Continuity of Structural Properties: No Dwarf/Giant Dichotomy}

The commonly held view that early-type "giant" $\left(M_{B} \lesssim-18\right)$ and "dwarf" $\left(M_{B} \gtrsim\right.$ -18) galaxies obey completely different photometric and structural scaling relations was introduced by Kormendy (1985) who argued that "there is a large discontinuity in the parameter correlations for bright [giant] ellipticals, including M32, and dwarf spheroidals [dwarfs]". However, it was noted by Jerjen \& Binggeli (1997) and Graham \& Guzmán (2003) that the Kormendy (1985) sample had a marked absence of galaxies with $M_{B} \sim$ -18 : the precise dividing point between the so-called dwarf and giant populations. In recent years, as high-quality photometric data have become available for more and more early-type galaxies, the reality of this dwarf/giant dichotomy has been questioned in a number of studies (see also early contributions from Binggeli, Sandage \& Terenghi 1984 and Caldwell 1983, 1987): e.g., Jergen \& Binggeli (1997), Jerjen, Binggeli \& Freeman (2000), Graham \& Guzmán (2003), Graham et al. (2003), Gavazzi et al. (2005), Aguerri et al. (2005), Zibetti et al. (2005), Côté et al. (2006), Ferrarese et al. (2006a). In this section, we present new evidence from the ACS Virgo and Fornax Cluster Surveys supplementing these data with structural parameters for additional faint galaxies in Virgo, Fornax and the Local Group - that there is a continuity in the photometric and structural properties of early-type galaxies for $M_{B} \gtrsim-20$. 

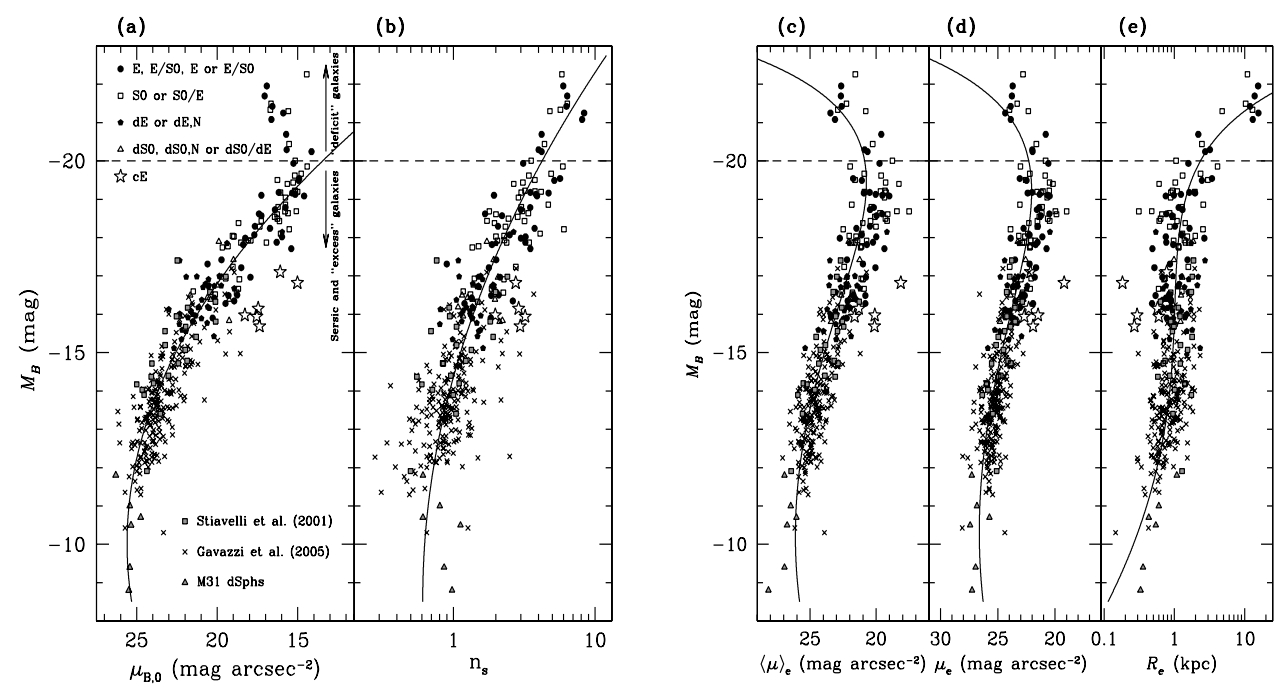

Figure 2. (Panel a) Relationship between absolute blue magnitude, $M_{B}$, and the intrinsic $B$-band central surface brightness for early-type galaxies from the ACS Virgo and Fornax Cluster surveys. Also shown are dE and E galaxies in the Virgo Cluster from Gavazzi et al. (2005), earlytype Virgo dwarfs from Stiavelli et al. (2001) and M31 dSphs from McConnachie \& Irwin (2006). The smooth curve shows the best-fit quadratic relation for galaxies fainter than $M_{B}=-20$ (indicated by the dashed line). (Panel b) Absolute blue magnitude plotted against Sérsic index, $\mathrm{n}_{s}$. The best-fit quadratic relation is again shown by the smooth curve. (Panel $c$ ) Dependence on $M_{B}$ of the average $B$-band surface brightness within the effective radius. (Panel $d$ ) Dependence on $M_{B}$ of the $B$-band surface brightness measured at the effective radius. (Panel $e$ ) Dependence on $M_{B}$ of the effective radius, $R_{e}$. The solid curves in panels $(c)-(e)$ show the expected relations for Sérsic models, beginning with the $M_{B}-\mu_{0}$ and $M_{B}-n_{s}$ relations shown in panels $(a)$ and $(b)$.

Panel ( $a$ ) of Fig. 2 shows the relationship between absolute blue magnitude, $M_{B}$, and central surface brightness, $\mu_{B, 0}$, for the Virgo and Fornax galaxies. $\dagger$ Absolute magnitudes have been derived using the SBF distances from Mei et al. (2007) and Blakeslee et al. (2008, in preparation). For bright galaxies $\left(M_{B} \lesssim-20\right)$, core-Sérsic models (Graham et al. 2003) have been used to fit the profiles. For galaxies fainter than $M_{B} \sim-20$, a Sérsic law is a good representation of the entire profile when a nucleus is not present, while the profiles of nucleated galaxies are fitted by adding a second Sérsic component for the nucleus. Thus, for nucleated galaxies, the central surface brightnesses shown in Fig. $2 a$ were measured using the inward extrapolation of the outer Sérsic component (i.e., excluding the central excess). Fig. $2 b$ shows the corresponding relationship between $M_{B}$ and Sérsic index, $\mathrm{n}_{s}$.

Also included in Fig. $2 a b$ are 23 early-type dwarf galaxies in Virgo and Fornax from Stiavelli et al. (2001). For consistency, we have re-fitted the WFPC2 profiles for these galaxies using the same family of models used for the ACS samples: i.e., single Sérsic models for non-nucleated galaxies and double Sérsic models for galaxies with nuclear excesses. At still lower luminosities, we plot the results of fitting Sérsic models to the brightness profiles of six M31 dSph galaxies from McConnachie \& Irwin (2006). In these cases, the surface brightness at $0.05^{\prime \prime}$ is measured after shifting the profiles to the distance of Virgo. Finally, we include 201 of the 226 early-type (i.e., dE or E) Virgo galaxies

$\dagger$ Measured at $R=0.05^{\prime \prime}$, equivalent to one ACS/WFC pixel, or $\approx 4-5$ pc at the distance of Virgo/Fornax. 
observed by Gavazzi et al. (2005) with the $2.5 \mathrm{~m}$ Isaac Newton Telescope, (i.e., omitting 25 galaxies that were already included in the ACS or WFPC2 samples).

The smooth curves in Fig. $2 a$ and $2 b$ show the best-fit quadratic relations for the combined sample. Since the "deficit" galaxies are thought to have central cores depleted by the dynamical evolution of supermassive black hole binaries, we have excluded these galaxies from the analysis. We have also excluded six Virgo or Fornax galaxies that have, at various times, been classified as members of the rare class of "compact ellipticals" (cEs): VCC1297, VCC1199, VCC1192, VCC1440, VCC1627 and FCC143 (indicated by the open stars in Fig. 2). Such galaxies are thought to have had their internal structure heavily modified by tidal interactions with nearby companions; indeed, all of these galaxies, except for VCC1440, are observed to have a bright, nearby companion with a similar velocity and SBF distance. These objects aside, we see from Fig. $2 a b$ that there is a clear, continuous sequence in both panels extending from $M_{B} \sim-20$ down to the level of the M31 dwarfs - a range of more than 11 mag.

Fig. $2 c b d$ shows three additional scaling relations for this same sample: the dependence on absolute blue magnitude of (1) mean surface brightness within the effective radius, $\langle\mu\rangle_{e} ;(2)$ surface brightness measured at the effective radius, $\mu_{e}$; and (3) the effective radius, $R_{e}$. Once again, fainter than $M_{B} \sim-20$, there is no discontinuity in any of these relations. Moreover, as noted by Graham \& Guzman (2003) the expected parameter correlations for Sérsic models can be calculated directly from the $M_{B}-\mu_{0}$ and $M_{B}-\mathrm{n}_{s}$ relations shown in Fig. 2ab. These relations are shown as the smooth curves in each panel. We conclude that there is no evidence for a "dwarf/giant dichotomy".

\section{The Extragalactic Distance Scale}

It is well known that the Virgo Cluster has a complex and irregular structure on the plane of the sky, spanning an area of $\approx 140 \mathrm{deg}^{2}$ (see Fig. 2 of Binggeli, Sandage \& Tammann 1985). The cluster's structure along the line of sight is, however, a matter of longstanding debate with estimates of the back-to-front depth differing wildly. At one extreme, Young \& Currie (1995) report a depth of $\pm 6-8 \mathrm{Mpc}$ by employing the shape of the brightness profiles for dwarf elliptical galaxies as a distance indicator; a similar estimate based on Virgo spirals has been reported by Yasuda, Fukugita, \& Okamura (1997) using the Tully-Fisher relation. On the other hand, measurements from the SBF method usually point to a much smaller depth (e.g., Neilsen \& Tsvetanov 2000; Jerjen, Binggeli \& Barazza 2004).

The ACSVCS was designed to provide accurate distances for our program galaxies using the $z$-band SBF method (see Fig. 7 of Côté et al. 2004). Details of our implementation of this technique were given in Mei et al. (2005ab) and, in Mei et al. (2007), Virgo's three-dimensional structure was studied using distances with a mean random uncertainty of $0.07 \mathrm{mag}(0.5 \mathrm{Mpc})$ for 84 galaxies. Apart from five galaxies located at a distance of $d \sim 23 \mathrm{Mpc}$ - which are thus members of the background $\mathrm{W}^{\prime}$ Cloud - we find the ACSVCS galaxies to have a narrow distribution around our adopted distance of $\langle d\rangle=16.5 \pm 0.1$ (random mean error) $\pm 1.1 \mathrm{Mpc}$ (systematic). The back-to-front depth of the cluster measured from our sample is $2.4 \pm 0.4 \mathrm{Mpc}$ (i.e., $\pm 2 \sigma$ of the intrinsic distance distribution). M87 and M49 - the dominant galaxies of the A and B subclusters that comprise the Virgo Cluster — were found to lie at distances of $16.7 \pm 0.2$ and $16.4 \pm 0.2$ $\mathrm{Mpc}$, respectively. Interestingly, there is some evidence for a weak correlation between velocity and line-of-sight distance that may be a faint echo of the cluster velocity distribution not having yet completely virialized. In three dimensions, the cluster has a 

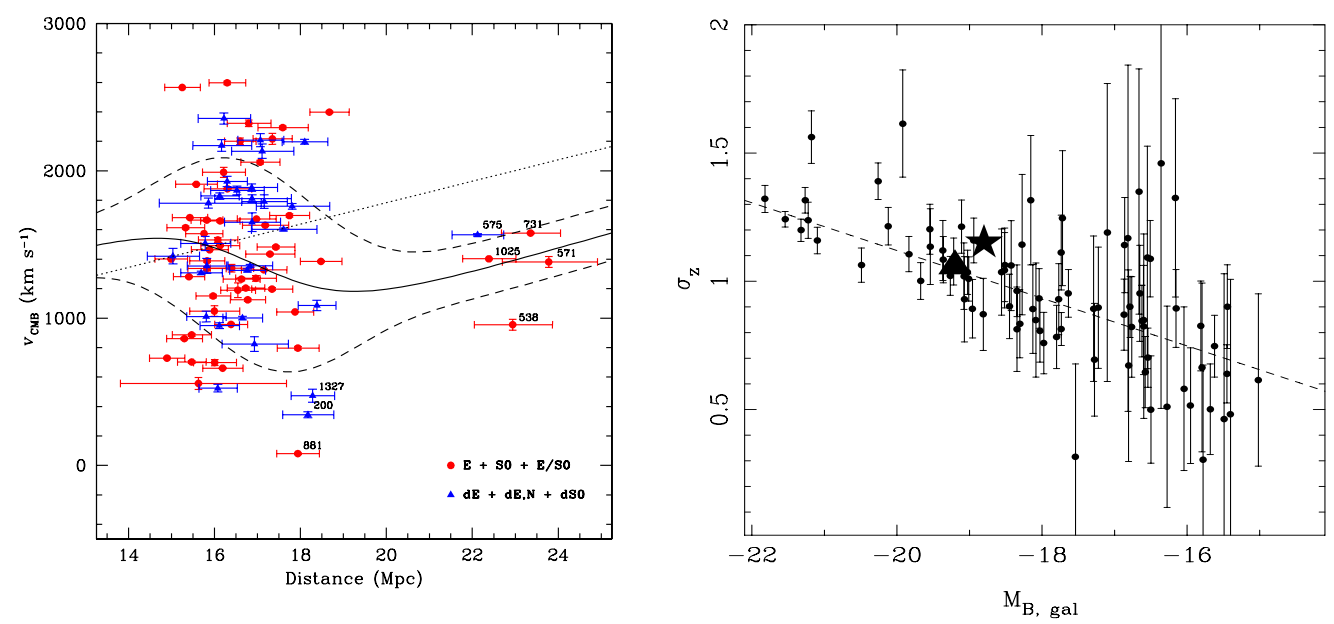

Figure 3. (Left) Velocity-distance relation for galaxies from the ACSVCS. Results for "giants" and "dwarfs" in the ACSVCS (as classified by Binggeli, Sandage \& Tammann 1985) are shown as the red circles and blue triangles, respectively. The dotted lines shows the undisturbed Hubble Flow in the direction of Virgo for an assumed Hubble Constant of $H_{0}=73 \mathrm{~km} \mathrm{~s}^{-1} \mathrm{Mpc}^{-1}$. The predicted distance-velocity relation for a line of sight passing through the cluster, based on the model of Tonry et al. (2000) for large-scale flows in the local universe, is shown by the solid (mean velocity) and dashed curves $( \pm 1 \sigma$ limits). Note the presence of five galaxies associated with the $\mathrm{W}^{\prime}$ Cloud at $d \approx 23 \mathrm{Mpc}$. See Mei et al. (2007) for details. (Right) Gaussian dispersion, $\sigma_{z}$, vs. galaxy absolute blue magnitude for the $z$-band globular cluster luminosity functions (GCLFs) of 89 ACSVCS galaxies. The GCLF width varies systematically with host luminosity, being narrower in fainter galaxies. The two outliers at $M_{B}=-21.2$ and -19.9 correspond to the galaxies VCC798 and VCC2095, both of which have large excesses of diffuse star clusters (see $\S 6$ and Peng et al. 2006b). The large star is plotted at the spheroid luminosity and GCLF dispersion of the Milky Way, while the large triangle marks the bulge luminosity and GCLF dispersion of M31. See Jordán et al. (2006, 2007b) for details.

slightly triaxial distribution, with axis ratios of (1:0.7:0.5) and a principal axis inclined by $\sim 20^{\circ}-40^{\circ}$ from the line of sight.

In Jordán et al. $(2006,2007 \mathrm{~b})$, the SBF distances were used to examine the universality of the globular cluster luminonsity function (GCLF) - long thought to be a standard candle useful in distance estimation (see Harris 2001 and references therein). Using a Gaussian parameterization of the GCLF, a highly significant correlation was found between GCLF dispersion, $\sigma_{z}$, and galaxy luminosity, in the sense that the globular cluster systems of fainter galaxies have narrower luminosity functions (see the right panel of Fig. 3). This behavior was found to be mirrored by a steepening of the globular cluster mass function for $\mathcal{M} \gtrsim 3 \times 10^{5} \mathcal{M}_{\odot}$, a mass regime in which the shape of the GCLF has probably not been strongly affected by dynamical evolution. In bright galaxies, the GCLF "turns over" at the canonical mass scale of $\mathcal{M}_{T O} \approx 2 \times 10^{5} \mathcal{M}_{\odot}$. However, the turnover was also shown to scatter to significantly lower values, $\mathcal{M}_{T O} \sim(1-2) \times 10^{5} \mathcal{M}_{\odot}$, in galaxies fainter than $M_{B} \gtrsim-18.5$ - an important consideration if the GCLF is to be applied as a distance indicator in "dwarf" galaxies.

Finally, we note that at the distance of the Virgo Cluster, 1 " corresponds to a projected distance of $\approx 80 \mathrm{pc}$. Thus, Galactic globular clusters, with typical sizes of $r_{h} \approx 3 \mathrm{pc}$, would be marginally resolved in our ACS images. As part of a customized data reduction pipeline (Jordán et al. 2004a), we measured photometric and structural parameters for candidate globular clusters by fitting the two-dimensional ACS surface brightness profiles with 
PSF-convolved isotropic, single-mass King (1966) models; i.e., for each object classified probabilistically (Peng et al. 2006a) as a globular cluster candidate we measure, in both the $g$ and $z$ bandpasses, the total magnitude, King concentration index, $c$, and half-light radius, $r_{h}$. Jordán et al. (2005) showed that the $r_{h}$ distribution has a characteristic form in the ACSVCS galaxies, with a peak at $\approx\left\langle r_{h}\right\rangle=2.7 \pm 0.35 \mathrm{pc}$ (after correcting for weak dependencies on galaxy and globular cluster color, and underlying galaxy surface brightness). Thus, it appears that the mean cluster half-light radius in early-type galaxies can be successfully used as a standard ruler for distance estimation, with an overall precision of $\sim 15 \%$.

\section{The Globular Cluster Systems of Early-Type Galaxies}

As the above discussion makes clear, a key objective of both the ACSVCS and ACSVCS is the study of thousands of globular clusters associated with the program galaxies. Indeed, the images are sufficiently deep that $\sim 90 \%$ of the globular clusters falling within the ACS fields can be detected at a high level of completeness (Côté et al. 2004). While the analysis of the globular cluster systems in Fornax is still in progress, we have reported our findings for Virgo in several papers that discuss the cluster colors/metallicities (Peng et al. 2006a), sizes (Jordán et al. 2005), luminosities/masses (Jordán et al. 2006, 2007b), formation efficiencies (Peng et al. 2007), their distribution in the color-magnitude plane (Mieske et al. 2006), and the connection to low-mass X-ray binaries (Jordán et al. 2004b; Sivakoff et al. 2007). The reader is referred to these papers for complete details on these issues. Here we focus on a single topic: the color/metallicity distributions of globular clusters in the ACSVCS (e.g., Peng et al. 2006a).

Somewhat surprisingly, the ACSVCS galaxies were found to have bimodal, or asymmetric, globular cluster color distributions at all luminosities (see the left panel of Fig. 4). Almost all galaxies in the survey were found to possess a population of metal-poor clusters, whereas the fraction of metal-rich clusters was found to increase steadily with galaxy luminosity, from $\sim 15 \%$ to $60 \%$ over the range $-22 \lesssim M_{B} \lesssim-15$. The colors of both subpopulations were found to correlate with host galaxy luminosity, with the red population having a steeper slope.

A key reason that the ACVCS and ACSFCS surveys were carried out in the F475W and F850LP filters is the excellent metallicity sensitivity afforded by the $(g-z)$ color index (see Fig. 4-6 of Côté et al. 2004). However, converting $(g-z)$ colors to metallicities presented something of a challenge since there was no empirical $(g-z)-[\mathrm{Fe} / \mathrm{H}]$ relation for old stellar populations available at the outset of the survey. Peng et al. (2006a) therefore presented a preliminary (and non-linear) $(g-z)-[\mathrm{Fe} / \mathrm{H}]$ relation based on CTIO $0.9 \mathrm{~m}$ telescope imaging of Galactic globular clusters, and supplemented with data for several dozen clusters in the Virgo ellipticals M49 and M87. This relation was used to convert the observed color distributions into metallicity distributions, with the interesting result that the metallicities of the metal-poor and metal-rich cluster were found to vary similarly with respect to the stellar mass, $\mathcal{M}_{*}$, of the host galaxy: $[\mathrm{Fe} / \mathrm{H}] \propto \mathcal{M}_{*}{ }^{0.2}$. Taken at face value, this would suggest a commonality in the conditions governing the formation of metal-poor and metal-rich globular clusters.

\section{New Families of Hot Stellar Systems}

As discussed in $\S 1$, the Virgo and Fornax surveys were planned with very specific science goals in mind, most notably a better understanding of the photometric and structural properties of the program galaxies and their surrounding globular clusters. Yet the 

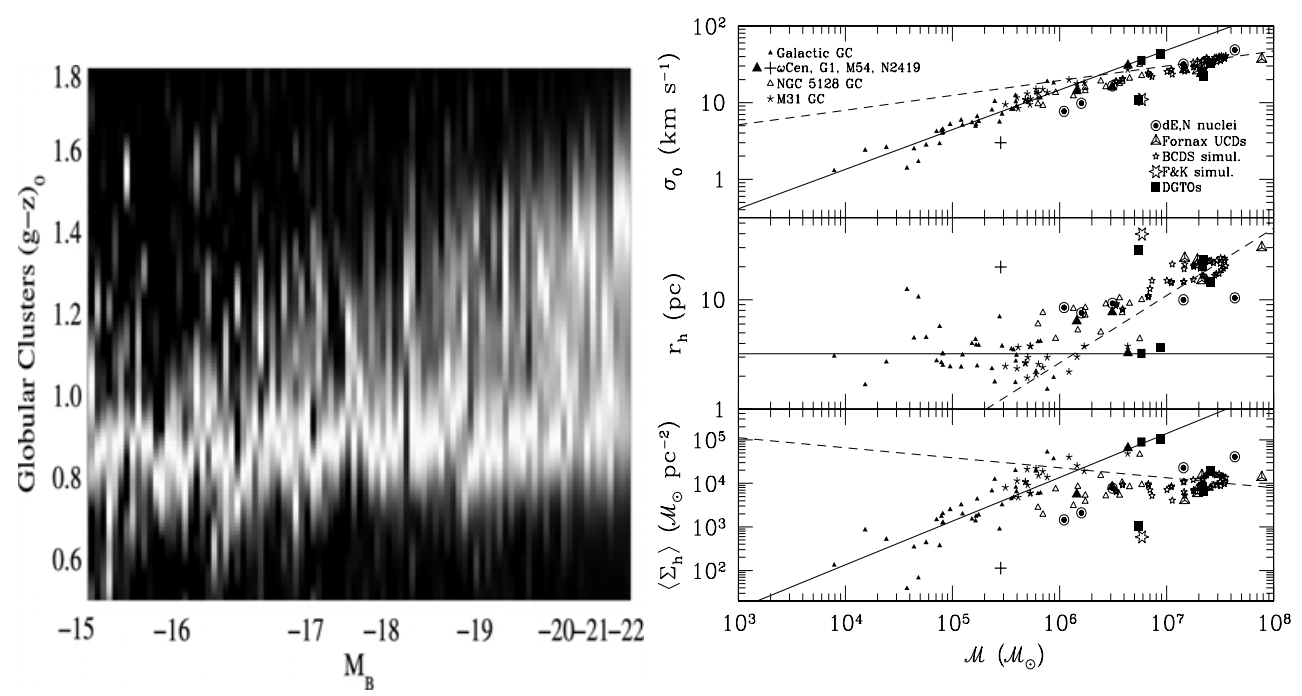

Figure 4. (Left) Globular cluster color distributions of ACSVCS galaxies ordered by absolute blue magnitude of the host galaxy. Each column in this image is a kernel density estimation of a galaxy's globular cluster color distribution, with white normalized to the peak density. The galaxies are ordered by luminosity, increasing from left to right. This image illustrates that all galaxies possess a population of blue clusters with similar color, while the color and relative fractions of red clusters is a strong function of galaxy luminosity. See Peng et al. (2006a) for details. (Right) Dynamical scaling relations for low-mass hot stellar systems: i.e., central velocity dispersion, half-light radius and mass surface density averaged within the half-light radius, are plotted against dynamical mass. The dashed line in each panel shows the downward extrapolation of the appropriate scaling relation for luminous elliptical galaxies. The solid lines in the top and bottom panels show the least-square fits to globular clusters in the Milky Way; in the middle panel, the solid line shows the median half-light radius of $r_{h}=3.2 \mathrm{pc}$ for Galactic globular clusters. Note the apparent transition between the two types of stellar systems (i.e., globular clusters and ultra-compact dwarf galaxies) at $\mathcal{M} \approx 2 \times 10^{6} \mathcal{M}_{\odot}$. See Haşegan et al. (2005) for details.

ACS images that form the basis of the surveys have proven rich datasets for studying new families of hot stellar systems that appeared serendipitously in the surveys: i.e., diffuse star clusters (DSCs, Peng et al. 2006b) and ultra-compact dwarf galaxies (UCDs) or, in our preferred nomenclature, dwarf-globular transition objects (DGTOs, Haşegan et al. 2005).

(1) Diffuse Star Clusters. Peng et al. (2006b) reported the detection of DSCs in twelve of the 100 ACSVCS program galaxies. Compared to globular clusters, DSCs have low luminosities $\left(M_{V} \gtrsim-8\right)$ and a broad distribution of sizes $\left(3<r_{h}<30 \mathrm{pc}\right)$. They are, however, principally characterized by their low mean surface brightnesses which can be more than $3 \mathrm{mag}$ fainter than a typical globular cluster $\left(\mu_{g} \gtrsim 20 \mathrm{mag} \operatorname{arcsec}^{-2}\right)$. Moreover, while the sizes of globular clusters are constant with luminosity, DSCs are bounded at the bright end by an envelope of nearly constant surface brightness. The median DSC colors $(1.1<g-z<1.6)$ are redder than metal-rich GCs and often as red as the galaxy itself. This suggests that most DSC systems thus have mean ages older than 5 Gyr or else have supersolar metallicities, implying that at least some DSCs are likely to be long-lived. In many respects, the DSCs therefore resemble the "faint fuzzies" identified in other nearby galaxies (e.g., Larsen \& Brodie 2000). The closest Galactic 
analogs to the DSCs appear to be the old open clusters, and if a DSC population did exist in the disk of the Milky Way, it would be very difficult to find.

(2) Ultra-Compact Dwarfs and/or Dwarf-Globular Transition Objects. UCDs are a potentially new class of stellar system first identified in spectroscopic surveys of the Fornax Cluster (Hilker et al. 1999; Drinkwater et al. 2000). In Haşegan et al. (2005), we studied the possible connection between globular clusters and UCDs by examining the photometric, structural and dynamical properties of compact objects having luminosities in the range $10^{6.25} \lesssim \mathcal{L}_{V} / \mathcal{L}_{V, \odot} \lesssim 10^{6.65}$ (DGTOs). Our principal finding from this analysis, which was based on an examination of the mass-based scaling relations for a sample of globular clusters and UCDs in the Virgo Cluster, the Local Group and NGC5128, was the detection of an apparent transition between these two types of stellar systems at a mass of $\mathcal{M} \approx 2 \times 10^{6}$ solar masses (see the right panel of Fig. 4). Furthermore, there is some evidence to suggest that either the presence of dark matter, or lengthy relaxation timescales, may distinguish UCDs from globular clusters. Five of the 13 DGTOs in Virgo were found to be associated with a single galaxy, M87, suggesting that proximity to the cluster center may be of critical importance for the formation of these rare objects. A detailed analysis of an expanded sample of DGTOs in Virgo and Fornax will be presented in Haşegan et al. (2008).

\section{Acknowledgements}

We thank Massimo Stiavelli and Alan McConnachie for kindly providing the brightness profiles for the low-luminosity galaxies in Virgo, Fornax and M31 discussed in $§ 3$.

\section{References}

Aguerri, et al. 2005, AJ, 130, 475

Binggeli, B., Sandage, A., \& Terenghi, M. 1984, AJ, 89, 64

Binggeli, B., Sandage, A., \& Tammann, G.A. 1985, AJ, 90, 1681

Caldwell, N. 1983, AJ, 88, 804

Caldwell, N. 1987, AJ, 94, 1116

Côté, P., et al. 2004, ApJS, 153, 223 (ACSVCS Paper I)

Côté, P., et al. 2006, ApJS, 165, 57 (ACSVCS Paper VIII)

Côté, P., et al. 2007, ApJ, in press (ACSFCS Paper II)

Drinkwater, M. J., et al. 2000, PASA, 17, 227

Ebisuzaki, T., Makino, J., \& Okumura, S.K. 1991, Nature, 354, 212

Faber, S. M., et al.1997, AJ, 114, 1771

Ferguson, H. C. 1989, $A J, 98,367$

Ferrarese, L., et al. 1994, AJ, 108, 1598

Ferrarese, L., et al. 2006a, ApJ, 164, 334 (ACSVCS Paper VI)

Ferrarese, L., et al. 2006b, ApJ, 644, L21

Ferrarese, L., et al. 2006c, arXiv:astro-ph/0612139

Ford, H. C., et al. 1998, Proc. SPIE, 3356, 234

Gavazzi, G., et al. 2005, A\& A, 430, 411

Graham, A. W., et al. 2003, AJ, 125, 2951

Graham, A. W., \& Guzmán, R. 2003, AJ, 125, 2936

Grant, N. I., Kuipers, J. A., \& Phillipps, S. 2005, MNRAS, 363, 1019

Harris, W. E. 2001, Saas-Fee Advanced Course 28: Star Clusters, 223

Haşegan et al. 2005, ApJ, 627, 203 (ACSVCS Paper VII)

Hilker, M., et al. 1999, A\&GAS, 134, 75

Jerjen, H., \& Binggeli, B. 1997, in ASP Conference Series, Vol. 116, p. 239

Jerjen, H., Binggeli, B., \& Freeman, K. C. 2000, AJ, 119, 593

Jerjen, H., Binggeli, B., \& Barazza, F. D. 2004, AJ, 127, 771 
Jordán, A., et al. 2004a, ApJS, 154, 509 (ACSVCS Paper II; J04)

Jordán, A., et al. 2004b, ApJ, 613, 279 (ACSVCS Paper III)

Jordán, A., et al. 2005, ApJ, 634, 1002 (ACSVCS Paper X)

Jordán, A., et al. 2006, ApJ, 651, L25.

Jordán, A., et al. 2007a, ApJS, 169, 213 (ACSFCS Paper I)

Jordán, A., et al. 2007b, ApJS, 171, 101 (ACSVCS Paper XII)

King, I. R. 1966, $A J, 71,64$

Kormendy, J. 1985, ApJ, 29573

Larsen, S. S., \& Brodie, J. P. 2000, AJ, 120, 2938

Lauer, T. R., et al. 1995, AJ, 110, 2622

Lauer, T. R., et al. 2007, ApJ, 664, 226

McConnachie, A. W., \& Irwin, M. J. 2006, MNRAS, 365, 1263

Mei, S., et al. 2005a, ApJS, 156, 113 (ACSVCS Paper IV)

Mei, S., et al. 2005b, ApJ, 625, 121 (ACSVCS Paper V)

Mei, S., et al. 2007, ApJ, 655, 144 (ACSVCS Paper XIII)

Mihos, J. C., \& Hernquist, L. 1994, ApJ, 437, L47

Neilsen, E. H., \& Tsvetanov, Z. I. 2000, ApJ, 536, 255

Peng, E. W. et al. 2006a, ApJ, 639, 95 (ACSVCS Paper IX)

Peng, E. W. et al. 2006b, ApJ, 639, 838 (ACSVCS Paper XI)

Peng, E. W. et al. 2007, ApJ, submitted (ACSVCS Paper XV)

Ravindranath, S., Ho, L. C., \& Filippenko, A. V. 2002, ApJ, 566, 801

Rest, A., et al. 2001, AJ, 121, 2431

Sérsic, J. -L. 1968, Atlas de Galaxias Australes (Córdoba: Obs. Astron., Univ. Nac. Córdova)

Sivakoff, G. R., et al. 2007, ApJ, 660, 1246

Stiavelli, M., et al. 2001, AJ, 121, 1385

Tonry, J. L., et al. 2000, ApJ, 530, 625

Yasuda, N., Fukugita, M., \& Okamura, S. 1997, ApJS, 108, 417

Young, C. K., \& Currie, M.J. 1995, MNRAS, 273, 1141

Zibetti, S., et al. 2005, in IAU Colloq. 198, p. 380 